\title{
Relative contact area in metal-polymer joints
}

\author{
Peter Ogar ${ }^{1, *}$, Yurij Alpatov ${ }^{1}$, and Denis Gorokhov ${ }^{1}$ \\ ${ }^{1}$ Russia Bratsk State University, 665709 Makarenko 40, Bratsk, Russia
}

\begin{abstract}
The paper is devoted to the specification of the parameters of Bartenev-Lavrentiev's empirical dependence for the relative area of contact with taking into account the mutual influence of asperities. The relative contact area is determined using a discrete roughness model for a wide range of distributions of the peaks of the asperities in the rough layer. It is shown that when determining the relative contact area, the distribution of the asperities in height practically does not affect the final result, since the obtained dependences coincide. This fact allowed us to determine the parameters of engineering dependence, which coincides with the results of theoretical studies.
\end{abstract}

\section{Introduction}

An important characteristic in determining the performance of joints of machine parts is the relative contact area. As follows from [1-3], as well as from the recent publications of the authors [4-7], for determining the relative contact area and especially with the use of lowmodulus materials, the mutual influence of microasperities should be taken into account. In these studies, using the discrete roughness model, analytical equations are given for determining the relative contact area as a function of the dimensionless force elastic geometric parameter $f_{y}$. At the same time, for practical calculations $[8,9]$, the empirical dependence of Bartenev-Lavrent'ev is widely used [10, p. 118]:

$$
\eta=1-\exp \left(-\beta \cdot\left(\frac{q_{c}}{E^{*}}\right)^{\chi}\right),
$$

where $\beta^{\bullet}$ is coefficient depending on the surface quality, in [8] the value $\beta^{\bullet}$ is determined analytically; $\chi=2 / 3$ (in paper [8] $\chi=1$ ); $q_{c}$ is the contour contact pressure, $E^{*}$ is the reduced modulus of elasticity.

It follows from [4-7] that without taking into account the mutual influence of the asperities, with using a discrete roughness model, the case is possible when $\eta=1$. This contradicts the data of [11-15] devoted to the study of almost full contact between rough surfaces.

\footnotetext{
${ }^{*}$ Corresponding author: ogar@brstu.ru
} 
The purpose of this paper is to refine the parameters of the empirical dependence (1), taking into account the latest trends in determining the relative contact area [4-7] with taking into account the mutual influence of the asperities.

\section{Model of a rough surface}

We use the discrete roughness model given in [4-7]. Microasperities can be represented as a set of identical spherical segments with a radius $r$, a base $a_{c}$ and a height $\omega R_{\max }$. The height distribution of the asperities corresponds to the bearing curve of the real surface profile, which is described by the regularized beta function:

$$
\eta(\varepsilon)=\frac{\mathrm{B}_{\varepsilon}(p, q)}{\mathrm{B}(p, q)}
$$

where $\mathrm{B}_{\varepsilon}(p, q), \mathrm{B}(p, q)$ are respectively incomplete and complete beta functions;

$$
p=\left(\frac{R_{p}}{R_{q}}\right)^{2}\left(\frac{R_{\max }-R_{p}}{R_{\max }}\right)-\frac{R_{p}}{R_{\max }}, \quad q=p\left(\frac{R_{\max }}{R_{p}}-1\right)
$$

$R_{p}, R_{q}, R_{\max }$ are the height parameters of roughness.

The density of the height distribution function of the asperities is described by the equation

$$
\varphi_{n}^{\prime}(u)=\frac{u^{p-2}(1-u)^{q-2}[(p-1)(1-u)-(q-1) u]}{\varepsilon_{S}^{p-1}\left(1-\varepsilon_{S}\right)^{q-1}},
$$

where $u$ is a distance from the level of peaks to the top of the asperities; $\varepsilon_{s}$ is determined from condition $\varphi_{n}\left(\varepsilon_{s}\right)=1 ; \omega=1-\varepsilon_{s}$.

Radius of the spherical segment is

$$
r=a_{c}^{2} /\left(2 \omega R_{\max }\right) .
$$

Figure 1 shows the density distribution of the asperities in height for the examples below of determining the relative contact area.

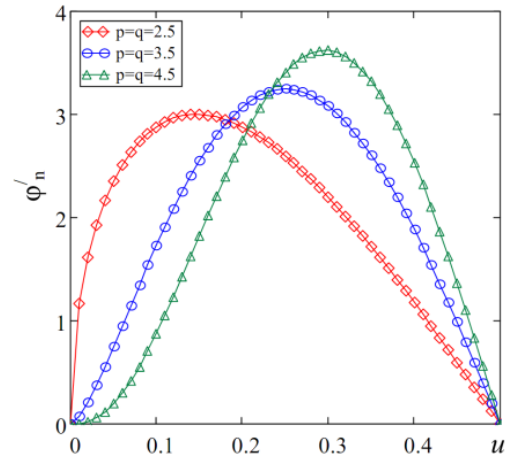

a)

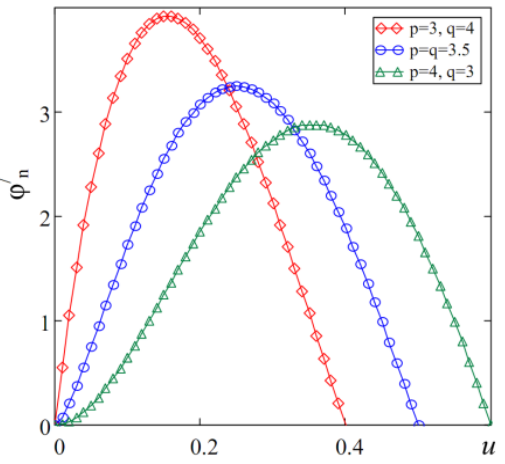

b)

Fig. 1. Density distribution of asperities for different values $p$ and $q$. 
It follows from Fig. 1, variation with the parameters $p$ and $q$ makes it possible to effectively control the distribution density of asperities in the height of the rough layer.

\section{Contact of a rigid rough surface with an elastic half-space.}

Below we give the system of equations [4-7], whose solution will allow us to determine the dependence of the relative area of contact $\eta$ on the dimensionless force elastic-geometric parameter $f_{y}$ which is determined by expression

$$
f_{q}=\frac{q_{c} a_{c}}{E^{*} \omega R_{\max }}
$$

where $E^{*}$ is the reduced modulus of elasticity.

To contact a rough surface with an elastic half-space, we have

$$
f_{q}(\varepsilon)=\frac{q_{c}(\varepsilon) a_{c}}{E^{*} \omega R_{\max }}=\frac{\frac{8}{3 \pi} \int_{0}^{\min \left(\varepsilon, \varepsilon_{s}\right)} \eta_{i}^{1,5} \varphi_{n}^{\prime}(u) d u}{1-\int_{0}^{\min \left(\varepsilon, \varepsilon_{s}\right)} \psi_{\eta}\left(\eta_{i}\right) \varphi_{n}^{\prime}(u) d u},
$$

where

$$
\begin{gathered}
\psi_{\eta}\left(\eta_{i}\right)=\frac{2}{\pi}\left[\arcsin \eta_{i}^{0.5}-\sqrt{\eta_{i}\left(1-\eta_{i}\right)}\right], \\
\eta_{i}=\frac{\varepsilon-u}{2 \omega}-f_{q}\left(1+\frac{f_{q}}{2}-\sqrt{\left(1+\frac{f_{q}}{2}\right)^{2}-\frac{\varepsilon-u}{2 \omega}}\right) .
\end{gathered}
$$

Relative contact area

$$
\eta(\varepsilon)=\int_{0}^{\min \left(\varepsilon, \varepsilon_{s}\right)} \eta_{i} \varphi_{n}^{\prime}(u) d u
$$

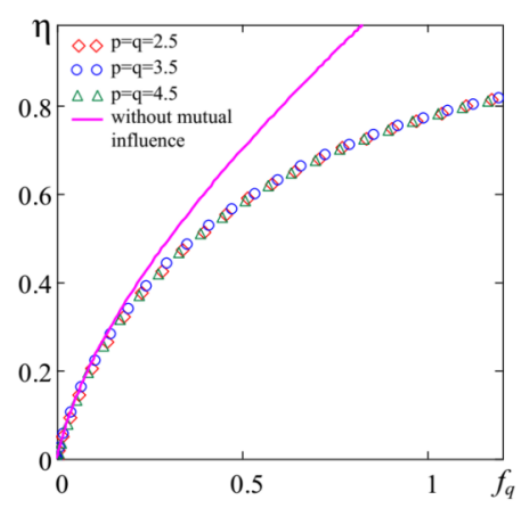

a)

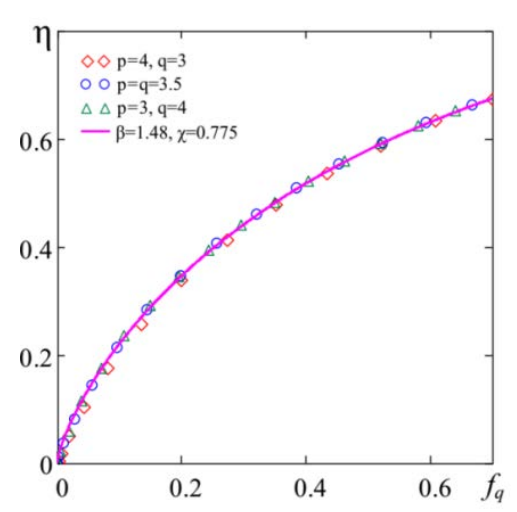

b)

Fig. 2. Dependence of the relative contact area on the dimensionless parameter $f_{y}$ : 
a) taking into account (dots) and without taking into account (the line) the mutual influence of asperities; b) for different values of the parameters $p$ and $q$.

To determine the dependence of $\eta\left(f_{q}\right)$, it is necessary to exclude parameter $\varepsilon$ from dependencies $f_{q}(\varepsilon)$ and $\eta(\varepsilon)$. Dependencies $\eta\left(f_{q}\right)$ for the distribution densities of the asperities corresponding to Fig. 1 are shown in Fig. 2 and are denoted by dots. The line in Fig. 2a a similar dependence is indicated without taking into account the mutual influence of the asperities.

It follows from Fig. 2, the dependences $\eta\left(f_{q}\right)$ for different values of the parameters $p$ and q practically merge into one line. Therefore, for engineering calculations, it is expedient to describe them by a single equation.

\section{Engineering method of calculating the relative area of contact.}

Using the technique of [10, p. 118], consider the contact of an individual asperity in the form of a spherical segment of radius $r$, base diameter $a_{c}$ and height $\omega R_{\max }$.

From the Hertz expressions [16], the radius of the contact area $a_{r}$ when the force $P$ is applied is given by

$$
a_{r i}=\left(\frac{3 P_{i} r}{4 E^{*}}\right)^{\frac{1}{3}}
$$

Relative contact area for a particular asperitiy

$$
\eta_{i}=\frac{a_{r}^{2}}{a_{c}^{2}}=\left(\frac{3 P_{i} r}{4 E^{*}}\right)^{\frac{2}{3}} \frac{1}{a_{c}^{2}}=\left(\frac{3}{8} \frac{P_{i}}{a_{c} E^{*} \omega R_{\max }}\right)^{\frac{2}{3}} .
$$

Taking into account that $q_{c}=P /\left(\pi a_{c}^{2}\right)$, we have

or

$$
\begin{gathered}
\eta_{i}=\left(\frac{3 \pi}{8} \frac{q_{c} a_{c}}{\omega R_{\max } E^{*}}\right)^{\frac{2}{3}} \\
\eta_{i}=\left(\frac{3 \pi}{8}\right)^{\frac{2}{3}}\left(f_{q i}\right)^{\frac{2}{3}}
\end{gathered}
$$

The only controlling parameter is $q_{c}$.

A differential equation which particular solution without taking into account the mutual influence of the asperities will be Eq. (13), has the form

$$
\frac{d \eta_{i}}{d f_{q}}=\frac{2}{3}\left(\frac{3 \pi}{8}\right)^{\frac{2}{3}}\left(f_{q}\right)^{-\frac{1}{3}} \Psi\left(\eta_{i}\right),
$$

where $\Psi\left(\eta_{i}\right)$ is function taking into account the mutual influence of asperities in their implementation. From physical representations, the function must satisfy the following conditions: in the region of low pressures $q_{c}$ (small values of $\left.f_{q}\right) \Psi\left(\eta_{i}\right)=1$; in the high- 
pressure region $q_{c}$ (large values of $\left.f_{q}\right) d \eta / d q_{c} \rightarrow 0\left(d \eta / d f_{q} \rightarrow 0\right)$, i.e. $\Psi\left(\eta_{i}\right)=0$. The simplest function satisfying these conditions is the function $\Psi\left(\eta_{i}\right)=1-\eta_{i}$. In this case, the solution of Eq. (14) for a single-level roughness model has the form

$$
\eta_{i}=1-\exp \left(-\beta f_{q}^{\chi}\right)
$$

where $\beta=(3 \pi / 8)^{\frac{2}{3}}=1,115, \chi=2 / 3$.

In Fig. $2 \mathrm{~b}$ points denote the analytical dependencies obtained as a result of the solution of the system of transcendental Eqs. (5) - (9). The solid line corresponds to the dependence

$$
\eta=1-\exp \left(-1,48 f_{q}^{0,775}\right) \text {. }
$$

It follows from Fig. 2b, the above approximation of the analytical dependencies describes them quite accurately and therefore can be recommended for engineering calculations of the relative contact area in metal-polymer compounds.

\section{Conclusion}

1. It is shown that when determining the relative contact area with taking into account the mutual influence of the asperities, their height distribution practically does not affect the final result, since all the obtained dependences on the dimensionless power elastic-geometric parameter $f_{y}$ practically coincide

2. This allowed us to accurately approximate the results of analytical studies and recommend for engineering calculations for elastic contact the universal dependence of the relative area of the dimensionless elastic elastic geometric parameter $f_{y}$.

\section{References}

1. I.G. Goryacheva, N.M. Dobychin, Contact problems in tribology (1988)

2. I.G. Goryacheva, Mechanics of frictional contact (2001)

3. A.P. Makushkin, Polymers in friction and seal assemblies at low temperatures (1993)

4. P.M. Ogar, D.B. Gorokhov, A.S. Kozhevnikov,. Contact problems in hermetic sealing studies, 242 (Brstu, Bratsk, 2017)

5. P.M. Ogar, D.B. Gorokhov, V.K. Elsukov, Systems. Methods. Technologies 2, 7 (2017)

6. P. Ogar, S. Belokobylsky, D. Gorokhov, V, Elsukov, Proc. of IX Int.Sci. Conf. BALTTRIB' 2017

7. P. Ogar, S. Belokobylsky, D. Gorokhov, Contact and Fracture Mechanics (2018)

8. A.I. Golubev, L.A. Kondakov, editors, Handbook of seals and sealing equipment (1994)

9. I.V. Kragel'skii, N.M. Dobychin, V.S. Kombalov, Basics of calculation for friction and wear (1974)

10. G.M. Bartenev, V.V. Lavrentiev, Friction and wear of polymers (1972)

11. S. Hyun, M.O. Robbins, Tribology Int., , 40, 1413 (2007)

12. C.-D. Yeo, R.R. Katta, J. Lee, A.A. Polycarpou, Tribology Int., 43, 1438 (2010)

13. Y. Xu, R.L Jackson, D..B. Marghitu, Int. J. Solids \& Structures, 51, 1075 (2014) 
14. V. A. Yastrebov 2 G. Anciaux, J.-F. Molinari, Int. J. Solids \& Structures, 52, 83 (2015)

15. Y. Xu, R.L Jackson, Tribology Int., 105, 274 (2017)

16. K.L. Johnson, Contact mechanics (1981) 